\title{
Comparison between SPH and MPS Methods for Numerical Simulations of Free Surface Flow Problems
}

\author{
Ahmed M. ABDELRAZEK ${ }^{1}$, Ichiro KIMURA ${ }^{2}$ and Yasuyuki SHIMIZU 3 \\ ${ }^{1} \mathrm{PhD}$. Student, Hydraulic Research Laboratory, Graduate School of Engineering, Hokkaido University (N13, \\ W8, Kita-ku, Sapporo 060-8628, Japan) \\ ${ }^{2}$ Member of JSCE, Dr. of Eng., Associate Professor, Hydraulic Research Laboratory, Graduate School of \\ Engineering, Hokkaido University (N13, W8, Kita-ku, Sapporo 060-8628, Japan) \\ ${ }^{3}$ Member of JSCE, Dr. of Eng., Professor, Hydraulic Research Laboratory, Graduate School of Engineering, \\ Hokkaido University (N13, W8, Kita-ku, Sapporo 060-8628, Japan)
}

\begin{abstract}
In this paper a comparison between two commonly mesh-free Lagrangian particles methods which have the ability to deal with problems with large free surface deformations i.e., weakly compressible smoothed Particle Hydrodynamic (SPH), and the original version of Moving Particle Semi-implicit (MPS) were done. Two benchmark tests, a collapse of a water column with a rigid obstacle, and dam break on a wet bed, are taken into consideration to examine the two methods. The results show that the particles from MPS are scattered and the water surface shape is scratchy, on the contrary the profile of water by SPH is commonly smooth and gentle. One of the advantages of SPH method is found that it yields rather smoother results even if the particles density becomes smaller. The CPU time for the presented cases with SPH is smaller than MPS, even though SPH requires smaller $\Delta \mathrm{t}$.
\end{abstract}

Key Words: SPH method, MPS method, free surface, particle method

\section{INTRODUCTION}

For a long time, the grid/mesh based numerical methods such as finite element method (FEM), finite volume methods (FVM), and finite difference methods (FDM) have been used as a major tool for computational fluid dynamics (CFD) problems with simple geometries. However, these methods suffer from difficulties in some aspects, such as dealing with complex geometries, deformable boundary, extremely large deformation and crack propagation. New types of numerical methods have been actively developed recently to solve CFD problems, called mesh-free particle (Lagrangian) methods. Smoothed particle hydrodynamics (SPH) method, and Moving Particle Semi-implicit (MPS) method are considered the most famous of these methods.

SPH method is a truly meshfree particle method and considered one of the oldest modern meshfree particle methods. It was originally invented for astrophysical applications ${ }^{1), 2)}$; then it has been extended and applied to a vast range of applications such as dynamic response of material strength ${ }^{3)}$, free surface fluid flows ${ }^{4}$, incompressible fluid flows ${ }^{5}$, multi-phase flows ${ }^{6}$, and turbulence flows ${ }^{7}$, etc.

In SPH method, each particle in the domain carries all field variable information like density, pressure, velocity and moves with the material velocity. The governing equations in the form of partial differential equations are converted to the particles equations of motion, and then they are solved by a numerical scheme.

MPS (Moving Particle Semi-implicit) method is introduced as a Lagrangian formulation for approximating the Navier-Stokes equations for incompressible fluids with free surface by Koshizuka and Oka $(1996)^{8), 9)}$. MPS method has been applied to many problems such as breaking wave problems ${ }^{10),}{ }^{11}$, fluid-structure interaction ${ }^{12)}$, and elastic bodies ${ }^{13)}$. Particle interaction models in MPS are prepared to replace the differential operators, such as gradient, divergence and Laplacian, then the governing equations are transformed into forms with interactions between moving particles. Many modifications and improvements have been proposed to MPS method ${ }^{10)}$ 14), 15), 16), but not considered in this paper.

The motivation of this paper is to compare between SPH and the standard MPS methods in simulations of free surface flow problems. Numerical simulations for two cases using the both methods are compared with experimental data in order to know which method is appropriate and more efficient. 


\section{SPH MODEL FOR WATER}

The governing equations of water in the framework of SPH consist of continuity and momentum conservation equations as follows:

$$
\begin{aligned}
& \frac{D \rho}{D t}=-\rho \frac{\partial v^{\alpha}}{\partial x^{\alpha}} \\
& \frac{D v^{\alpha}}{D t}=\frac{1}{\rho}\left(\frac{\partial \sigma^{\alpha \beta}}{\partial x^{\alpha}}\right)+f^{\alpha}
\end{aligned}
$$

where $\alpha$ and $\beta$ denote the Cartesian components $x, y$ and $z$ with the Einstein convention applied to repeated indices; $\rho$ is the density; $v$ is the velocity; $\sigma^{\alpha \beta}$ is the total stress tensor, $f^{\alpha}$ is the component of acceleration caused by external force, which is the gravity force in this study and $D / D t$ is the material (total) derivative, which is defined by:

$$
\frac{D}{D t}=\frac{\partial}{\partial t}+v^{\alpha} \frac{\partial}{\partial x^{\alpha}}
$$

SPH is based on interpolation theory. The governing equations, in the form of partial differential equation, are transformed into integral equations through the use of an interpolation function that gives the weight (kernel) estimation of the field variable at a point ${ }^{17}$. For any given functions $f(x)$, the "kernel estimation" of this function is defined as:

$$
\langle f(x)\rangle=\int_{\Omega} f\left(x^{\prime}\right) W\left(x-x^{\prime}, h\right) d x^{\prime}
$$

where $\Omega$ is the volume of the integration that contains $x$ and $x$; and $h$ is the smoothing length defining the influence domain of the "kernel estimate". $W(x-x, h)$ is the kernel, or smoothing function. There are many possible type of the smoothing function, which can satisfy the normalization condition, delta function property, and the compact condition ${ }^{17}$. The most famous one is the cubic spline interpolation functions, which was proposed by Monaghan and Lattanzio ${ }^{18)}$, defined as:

$$
W(R, h)=\alpha_{d} \times\left\{\begin{array}{cl}
1.5-R^{2}+0.5 R^{3} & 0 \leq R<1 \\
(2-R)^{3} / 6 & 1 \leq R<2 \\
0 & R \geq 2
\end{array}\right.
$$

where $\alpha_{d}=\frac{15}{7 \pi h^{2}}$ in 2D space, and $R=\frac{\left(x-x^{\prime}\right)}{h}$.

The stress tensor, $\sigma^{\alpha \beta}$, normally consists of two parts: an isotropic pressure $P$ and a viscous shear stress, which is proportional to the shear strain rate denoted by $\varepsilon$ through the viscosity $\mu$,

where:

$$
\sigma^{\alpha \beta}=-P \delta^{\alpha \beta}+\mu \varepsilon^{\alpha \beta}
$$

$$
\varepsilon^{\alpha \beta}=\frac{\partial v^{\alpha}}{\partial x^{\beta}}+\frac{\partial v^{\beta}}{\partial x^{\alpha}}-\frac{2}{3}\left(\frac{\partial v^{\gamma}}{\partial x^{\gamma}}\right) \delta^{\alpha \beta}
$$

Using the SPH approximation discussed and summarized in many literatures and text books ${ }^{17), 19)}$, the system of partial differential equations (1) and (2) can be converted into the SPH formulations which will be used to solve the motion of fluid particles as follows:

$$
\begin{aligned}
& \frac{D \rho_{i}}{D t}=\sum_{j=1}^{N} m_{j}\left(v_{i}^{\alpha}-v_{j}^{\alpha}\right) \frac{\partial W_{i j}}{\partial x_{i}^{\alpha}} \\
& \frac{D v_{i}^{\alpha}}{D t}=\sum_{j=1}^{N} m_{j}\left(\frac{\sigma_{i}^{\alpha \beta}}{\rho_{i}^{2}}+\frac{\sigma_{j}^{\alpha \beta}}{\rho_{j}^{2}}\right) \frac{\partial W_{i j}}{\partial x_{i}^{\beta}}+f^{\alpha}
\end{aligned}
$$

Similarly, the SPH approximation of shear strain rate $\varepsilon_{i}^{\alpha \beta}$ for particle $i$ is

$$
\begin{aligned}
\varepsilon_{i}^{\alpha \beta}=\sum_{j=1}^{N} \frac{m_{j}}{\rho_{j}} & \left(v_{i}^{\alpha}-v_{j}^{\alpha}\right) \frac{\partial W_{i j}}{\partial x_{i}^{\beta}}+\sum_{j=1}^{N} \frac{m_{j}}{\rho_{j}}\left(v_{i}^{\beta}-v_{j}^{\beta}\right) \frac{\partial W_{i j}}{\partial x_{i}^{\alpha}} \\
& -\left(\frac{2}{3} \sum_{j=1}^{N} \frac{m_{j}}{\rho_{j}}\left(v_{i}^{\gamma}-v_{j}^{\gamma}\right) \frac{\partial W_{i j}}{\partial x_{i}^{\gamma}}\right) \delta^{\alpha \beta}
\end{aligned}
$$

In order to solve Navier-Stokes equations for water the "equation of state" is used to estimate the pressure change of water. Following Monaghan" the pressure can be calculated using the equation of Tait:

$$
P=B\left[\left(\frac{\rho}{\rho_{o}}\right)^{\lambda}-1\right]
$$

where $\lambda$ is a constant $(=7), \rho_{o}$ is the reference density, $B$ is a problem dependent parameter, which sets a limit for the maximum change of the density and will be calculated as

$$
B=\frac{100 V_{\text {type }}^{2} \rho_{o}}{\lambda}, \quad V_{\text {type }}=\sqrt{2 \mathrm{gH}}
$$

where $V_{\text {type }}$ the typical speed of water and $H$ is the depth of the water.

To prevent shock waves and the penetration of particles, an artificial viscosity has been introduced to the pressure term in the momentum equation. The most widely used type is proposed by Monaghan ${ }^{18)}$ and specified as follows

$$
\pi_{i j}= \begin{cases}\frac{-\alpha \bar{c}_{i j} \phi_{i j}+\beta \phi_{i j}^{2}}{\bar{\rho}_{i j}} & v_{i j} . x_{i j}<0 \\ 0 & v_{i j} . x_{i j} \geq 0\end{cases}
$$

in which $\alpha$ and $\beta$ are constants and are taken 0.01, 1.0 respectively, and $c$ represent the speed of sound. As well as having beneficial effects, artificial viscosity can also introduce unwanted numerical defects in some cases, among which the excess dissipation and false shearing torque in rotating flows 20)

\section{MPS MODEL FOR WATER}

If the particles are moving with a velocity equal $v$, and density equal $\rho$, the continuity and momentum equations for incompressible flow are:

$$
\frac{D \rho}{D t}=0
$$




$$
\frac{D v}{D t}=-\frac{1}{\rho} \frac{\partial p}{\partial x_{i}}+v \nabla^{2} v+\mathrm{g}
$$

where $v$ is the kinmatic viscosity; and $\mathrm{g}$ is the acceleration of the body force. In MPS method, the governing equations are transformed to particles interactions equations, which describe the particles interact with its neighbors covered with a weight function $w\left(r, r_{e}\right)$, where $r$ is the distance between two particles, $r_{e}$ the radius of the interaction area. There are many types of weight (kernel) function, and the commonly used one for viscid, incompressible flow is proposed by Koshizuka et.al ${ }^{8}$,

$$
w(r)= \begin{cases}\frac{r_{e}}{r}, & r<r_{e} \\ 0, & r \geq r_{e}\end{cases}
$$

The particle number density for particle $i$, is defined using the kernel function as:

$$
\langle\mathrm{n}\rangle_{k}=\sum_{j \neq i} w\left(\left|r_{j}-r_{i}\right|\right)
$$

Based on the interpolation theory the gradient model and Laplacian model are expressed as ${ }^{11)}$,

$$
\begin{gathered}
\langle\nabla P\rangle_{i}=\frac{d}{n^{o}} \sum_{j \neq i}\left[\frac{P_{j}-P_{i}}{\left|r_{j}-r_{i}\right|^{2}}\left(r_{j}-r_{i}\right) w\left(\left|r_{j}-r_{i}\right|\right)\right] \\
\left\langle\nabla^{2} v\right\rangle_{i}=\frac{2 d}{\lambda n^{o}} \sum_{j \neq i}\left[\left(v_{j}-v_{i}\right) w\left(\left|r_{j}-r_{i}\right|\right)\right]
\end{gathered}
$$

where $d$ is the number of space dimensions, in this paper $d=2, n^{o}$ is the particle number density which is constant and obtained from the initial conditions; $\lambda$ is a coefficient introduced here so that the variance increase is equal to the analytical solution and given as:

$$
\lambda=\frac{\sum_{j \neq i}\left[w\left(\left|r_{j}-r_{i}\right|\right)\left|r_{j}-r_{i}\right|^{2}\right]}{\sum_{j \neq i}\left[w\left(\left|r_{j}-r_{i}\right|\right)\right]}
$$

\section{BOUNDARY CONDITIONS}

In SPH method, we use a dynamic boundary condition to represent the boundary particles, which are forced to follow the governing equations (continuity, momentum and state equations), but they are fixed. When the fluid particles are close to the boundary, the density of the boundary particles increase according the continuity equation which leads to increase in the pressure following the equation of state. Therefore the force exerted on the incoming fluid particles increases due to the pressure term in the momentum equation by generation of repulsion between the fluid and boundary particles [Dalrymple 2000] ${ }^{21)}$. While in MPS method we used the same type of boundary conditions in the original MPS method [Koshizuka $1998]^{12)}$.The solid wall (boundary) is represented with three layers of fixed particles. Velocities are always zero in those three layers and pressure is calculated on the first inner layer only to repulse the fluid particles from the wall. The outer two layers are represented to keep the particle number density $n^{o}$, at the vicinity of the walls.

\section{DIFFERENCE IN CALCULATION}

The differences between SPH and MPS methods can be summarized in the following points:

1- SPH is an explicit method, deals with water as an artificial fluid which is more compressible than the real one, and the pressure is calculated based on the equation of state, while MPS is an implicit method that deals with incompressible fluids, and the pressure is calculated from the Poisson equation.

2- The gradient of a function in SPH method is calculated using the gradient of smoothing function, $\left(\partial W_{i j} / \partial x_{i}\right)$, but in MPS method, the gradient of a function is calculated using algebraic operators with the weight function, $w\left(\left|r_{j}-r_{i}\right|\right)$ not using its gradient.

3- MPS has independence of parameters that usually require appropriate tuning, e.g. coefficients of artificial viscosity and XSPH scheme in the SPH method which in the practical viewpoint gives MPS superiority.

4- The comparison in this study is limited and it has under certain conditions. Table 1 shows the terms used in each method.

\section{VALIDATION AND APPLICATIONS}

Two benchmark tests have been considered to assess the performance of the two numerical methods in simulation of free surface flows, to show the models capabilities and to stand on the difference between the two models. A collapse of a water column with a rigid obstacle ${ }^{22)}$, and dam break on wet bed ${ }^{23)}$ are used.

\section{(1) Case 1: Collapse of water column with a rigid obstacle.}

Figure 1 shows a rectangular tank of $0.584 \mathrm{~m}$ wide contains a column of water with $0.146 \mathrm{~m}$ wide and $0.292 \mathrm{~m}$ high, a rigid obstacle is located in the

Table 1 Differences in the used methods

\begin{tabular}{|c|c|c|}
\hline & SPH & MPS \\
\hline Numerical Stabilizer & Exist (AV) & Not Exist \\
\hline Velocity smoothing & Exist (XSPH) & Not Exist \\
\hline Type of B.C & Dynamic B.C & $\begin{array}{c}\text { Original MPS } \\
\text { B.C }\end{array}$ \\
\hline
\end{tabular}




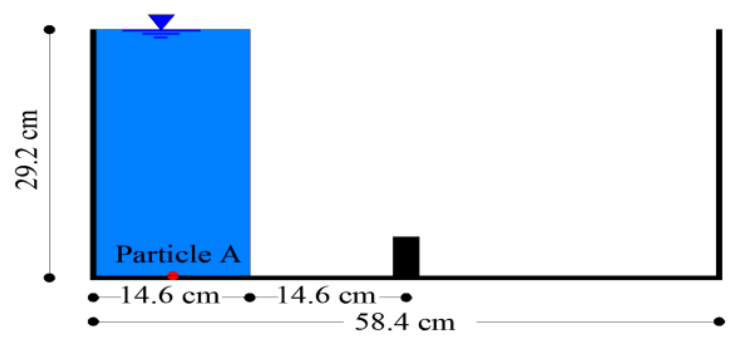

Fig. 1 Initial geometry, location of the rigid obstacle, and location of point (A)

middle of the bottom of the tank. This experiment was done by Koshizuka ${ }^{22}$. The number of fluid particles used in this simulation is 10658 with 0.002 $\mathrm{m}$ initial distance between particles. The gravitational force acts downwards with $\mathrm{g}=9.81$ $\mathrm{m} / \mathrm{s}^{2}$. Density and viscosity are respectively equal to $1000 \mathrm{~kg} / \mathrm{m}^{3}$ and $10^{-6} \mathrm{~m}^{2} / \mathrm{sec}$.

\section{(2) Case 2: Dam break on wet bed}

This experiment was performed by Janosi et al. $(2004)^{23)}$. Figure 2 shows a schematic sketch of the experimental setup. Water column with depth equal $0.15 \mathrm{~m}$ and width equal $0.38 \mathrm{~m}$ is retained behind a gate; the initial downstream water depth is $0.018 \mathrm{~m}$. In total 32250 fluid particles with an initial distance $0.002 \mathrm{~m}$ are used.

\section{RESULTS AND ANALYSIS}

Figures 3 and 4 present some snapshots of the position of the particles, and free-surface shape together with the pressure field (Figure 3) and velocity field (Figure 7) at different times using SPH and MPS methods compared with the experimental results. It is clear that the two methods can qualitatively simulate the free surface problems and capture the water free surface shape when we use a suitable number of particles. However the particle distributions in MPS method is more scattered than SPH method. The causes of these differences are that the particles in SPH method move using the XSPH variant (Monaghan, 1989) ${ }^{24)}$, which makes its movement more smooth, however, one must be careful when choosing XSPH parameters because it can give inaccurate results in some cases like sharp velocity gradients ${ }^{25)}$.

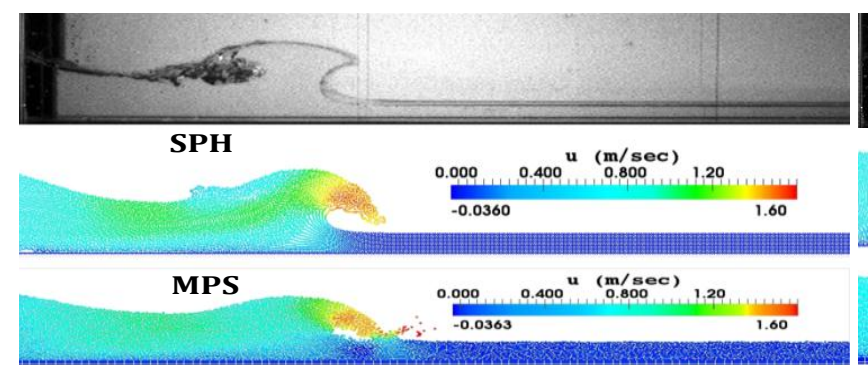

(A) $\mathrm{T}=0.281 \mathrm{sec}$

Fig. 4- Snapshots of water particles together with the velocity field at $(A) t=0.281$ and $(B) t=0.561$ sec, numerical results (SPH \&MPS) compared with experimental - case2

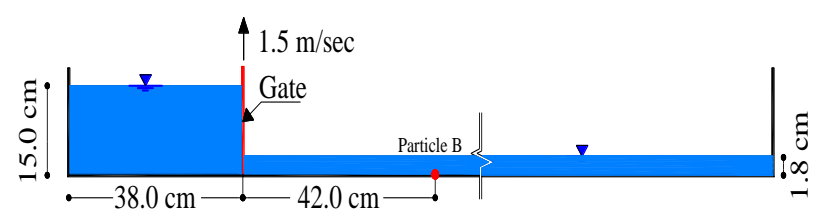

Fig. 2 Experimental setup of the dam breaks on a wet bed, and location of point (B)

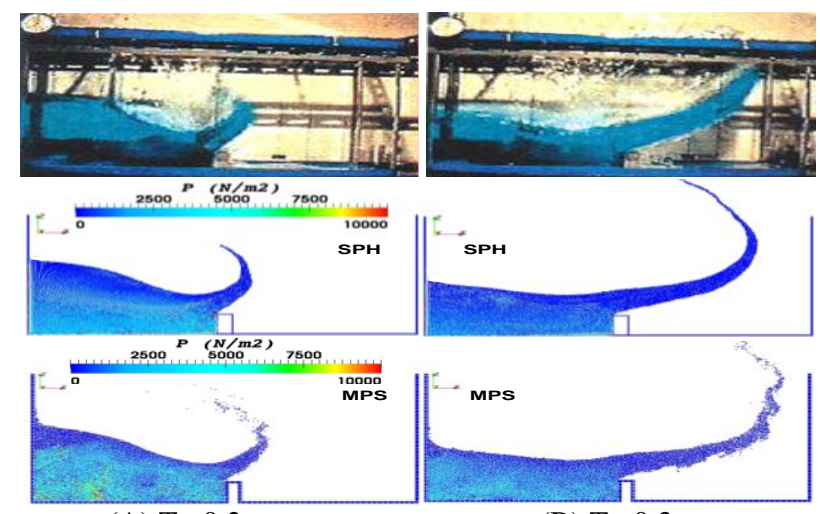

(A) $\mathrm{T}=0.2 \mathrm{sec}$

(B) $\mathrm{T}=0.3 \mathrm{sec}$

Fig. 3- snapshots of water particles together with the pressure field at (A) $\mathrm{t}=0.2 \mathrm{sec}$, (B) $\mathrm{t}=0.3 \mathrm{sec}$, numerical results ( $\mathrm{SPH}$ \&MPS) compared with experimental - case1.

In other hand at MPS method when $r=0$, the weight function, $w(r)$ will equal infinity. As a result of this, when two adjacent particles are close to each other, a rapid increase in the particle number density, $\langle\mathrm{n}\rangle_{k}$ happens and leads to increase in the pressure, and thus repulsion between particles occurs. Another important reason which gives scattered particles in MPS method is the numerical oscillation of pressure with high frequencies, which came from the source term of the Poisson equation. Figure 5 shows the values of pressure at wall particle (A) in case 1 and particle (B) in case 2. It is clear that the pressure from MPS method oscillates with severe fluctuation compared with SPH. Of course more accurate and stabilized simulation and less fluctuating pressure field would be obtained if we use the new improvements recently happened to the MPS method which include: introducing of a higher order source term in the Poisson equation ${ }^{16)}$ or considering a slight level of compressibility ${ }^{26}$, etc., and we will consider that in the future studies.

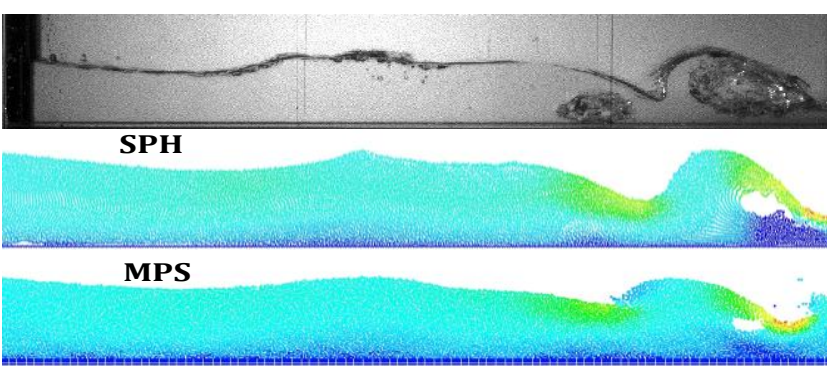

(B) $\mathrm{T}=0.561 \mathrm{sec}$ 


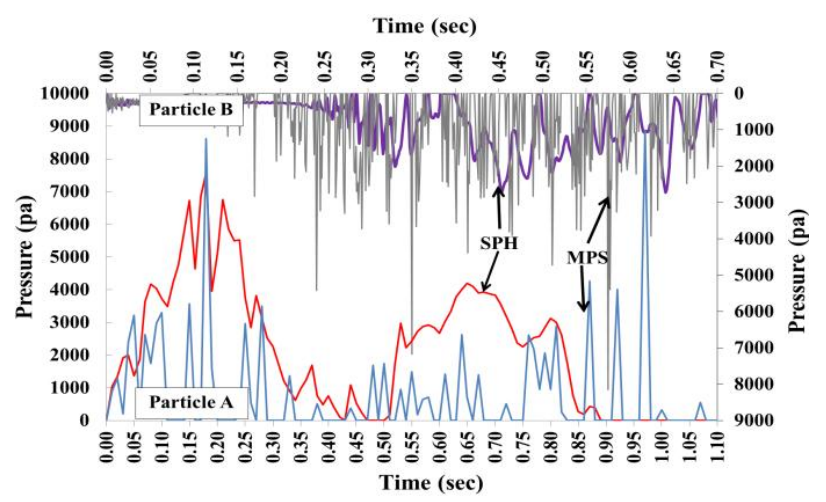

Fig. 5- Temporal variations of pressure at particles (A) and (B)

\section{1) Effect of the initial particles spacing:}

In order to investigate the convergence in this study and to know the effect of the initial particle spacing on the numerical results, three different initial particle spacings were selected in each case which are $0.008 \mathrm{~m}, 0.004 \mathrm{~m}$, and $0.002 \mathrm{~m}$. From Figures 6 and 7, it is obvious that increasing the particles spacing decreases the number of fluid particles involving in the simulations, which leads to an inaccurate simulation and inconsistent shape of the free surface with the experimental results. Also in SPH method, if we use a suitable number of particles; not dense nor coarse; It gives comparatively smooth results and consistent free surface shape, unlike MPS method needs dense particles to capture the details of flow, but still the problem of scattered particles in MPS exists

Also we can notice from figures 6 and 7 in the SPH results, as the distance between particles increases the particles move away from the boundaries noticeably. Might be the reason is using the dynamic boundary condition type.

\section{2) Computational efficiency}

In order to know which method is more efficient, we have to consider the simulation time elapsed for each case. Table 2, shows the elapsed time to simulate the first and last steps in the calculation of case 2,(from 0.00 to 0.001 and from 0.699 to 0.700 ). It is clear that the difference between the two

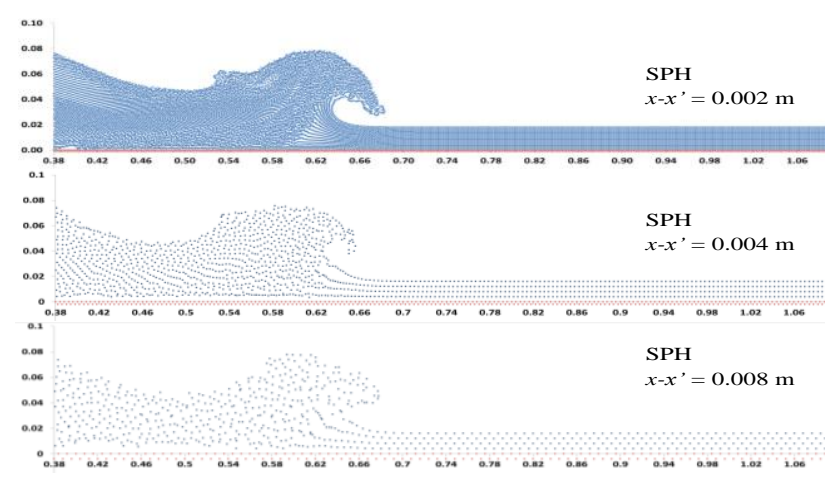

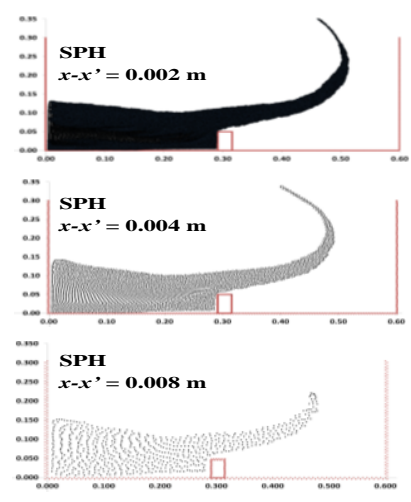

(A) SPH Method

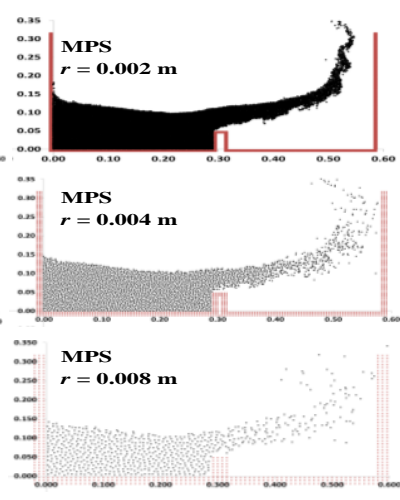

(B) MPS Method

Fig. 6 Particles positions and free surface shape using various initial particle spacing at $\mathrm{t}=0.3 \mathrm{sec}$ (case 1 )

elapsed times in MPS method are bigger than the difference in SPH method, because in MPS method the pressure calculation using Poisson equation takes time at the beginning of the simulation, but after the wave breaks the pressure calculations time is reduced. In case of using $0.002 \mathrm{~m}$ spacing between fluid particles, the first step calculation is 2.33 times larger than the last step in MPS, while in SPH almost no difference, leading to increase the total computation time in MPS method. Table 3 shows the total time elapsed in hour to simulate case 1 to reach $1.1 \mathrm{sec}$, and case 2 to reach $0.7 \mathrm{sec}$. We can notice that the total computational time using the SPH method is 1.98 times smaller than MPS method in the first case and 5.41 times in the second case when we used $0.004 \mathrm{~m}$ spacing between fluid particles. These differences become 4.37 times smaller in case 1 and 3.22 times in case 2 using $0.002 \mathrm{~m}$ spacing between fluid particles.

Table 2 First and last CPU time step (sec.) - case 2

\begin{tabular}{|c|c|c|c|c|c|c|}
\cline { 2 - 7 } & \multicolumn{3}{c|}{ SPH method } & \multicolumn{3}{c|}{ MPS method } \\
\cline { 2 - 7 } & \multicolumn{2}{c|}{$\begin{array}{c}\text { Distance between } \\
\text { Particles (m) }\end{array}$} & \multicolumn{3}{c|}{$\begin{array}{c}\text { Distance between } \\
\text { Particles }(\mathrm{m})\end{array}$} \\
\cline { 2 - 7 } & 0.008 & 0.004 & 0.002 & 0.008 & 0.004 & 0.002 \\
\hline $\begin{array}{c}1^{\text {st }} \mathrm{CPU} \\
\text { time step }\end{array}$ & 0.359 & 2.262 & 19.81 & 2.663 & 7.119 & 27.387 \\
\hline $\begin{array}{c}\text { Last CPU } \\
\text { time step }\end{array}$ & 0.218 & 1.929 & 19.80 & 0.499 & 3.039 & 11.721 \\
\hline
\end{tabular}

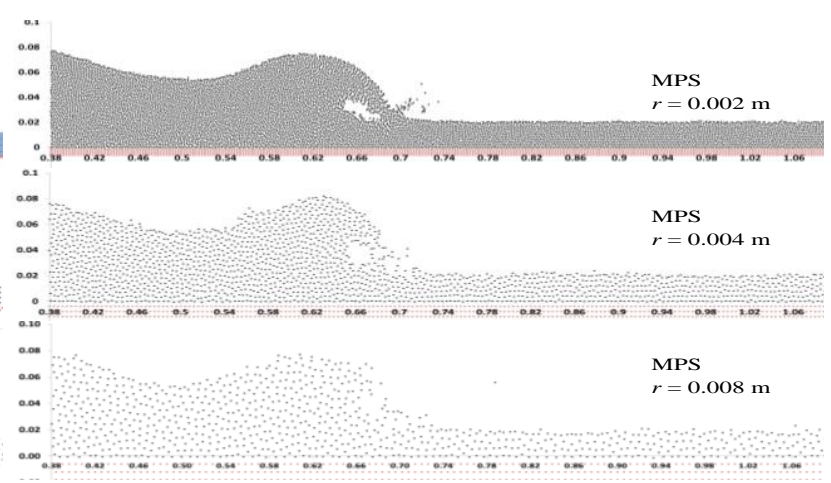

Fig. 7 Particles positions and free surface shape using various initial particle spacing at $t=0.281 \mathrm{sec}$. 
Table 3 Total time elapsed (hr.)

\begin{tabular}{|l|c|c|c|c|}
\cline { 2 - 5 } \multicolumn{1}{c|}{} & \multirow{2}{*}{ Method } & \multicolumn{3}{|c|}{ Distance between Particles $(\mathrm{m})$} \\
\cline { 2 - 5 } \multicolumn{1}{c|}{ Case 1 } & & 0.008 & 0.004 & 0.002 \\
\cline { 2 - 5 } & SPH & 0.112 & 0.794 & 6.069 \\
\hline \multirow{2}{*}{ Case 2 } & MPS & 0.139 & 1.572 & 26.526 \\
\cline { 2 - 5 } & SPH & 0.068 & 0.488 & 6.819 \\
\cline { 2 - 5 } & MPS & 0.250 & 2.640 & 21.996 \\
\hline
\end{tabular}

\section{CONCULSION}

The paper presents a comparative study between SPH and the standard MPS methods which are mesh free, lagrangian, particle methods. The numerical results for a collapse of water column with a rigid obstacle and a dam break on wet bed were compared with the experimental results. The results showed that the two methods can deal with problems with large deformations of the water free surface. The particles distribution from the SPH method is more stable than MPS method, due to using the artificial viscosity, and the predicted free surface from SPH calculation is smoother than the free surface from MPS calculation which gives noisy and splashing free surface. An advantage of SPH method is that even if rather a coarse particles arrangement, it yields relatively smooth results and reduces the computation time. One of the features of using MPS method, it has independence upon parameters that usually require appropriate tuning unlike the SPH method. We only consider MPS method in original form. Recently, many studies have been done to improve MPS, in particular, correcting unreasonable pressure oscillations. In the next step, we want to consider such refined MPS methods to clarify such refinement effects and their advantages compared with SPH, also using other types of boundary conditions like repulsive boundary condition with the SPH method to stand on which type is better, and taking into consideration one of the important aspects which is compared in terms of 'volume conservation'.

\section{ACKNOWLEDGMENT:}

The first author would like to thank MEXT for the Japanese government (Monbusho) scholarship.

\section{REFERENCES}

1) Lucy L.: A numerical approach to testing the fission hypothesis, Astronomical J., Vol.82, pp.1013-1024, 1977.

2) Gingold R.A., Monaghan J.J.: Smoothed particle hydrodynamics: theory and application to nonspherical stars, Mon.Not.Roy.Astron.Soc, Vol.181, pp.375-389, 1977.

3) Libersky LD, Petschek AG.: Smoothed particle hydrodynamics with strength of materials, In Proceedings of the Next Free Lagrange Conference, Vol. 395, Springer: New York, pp.248-257, 1991.

4) Monaghan J.J.: Simulating free surface flows with SPH, J. Comput. Phys., Vol.110, pp.399-406, 1994.
5) Cummins SJ, Rudman M.: A SPH projection method, J.Comput.Phys., Vol.152, pp.584-607, 1999.

6) Monaghan J.J., Kocharyan A.: SPH simulation of multiphase flow, comput. phys. commun., Vol.87, pp.225-235, 1995.

7) Monaghan J.J.: SPH compressible turbulence, Mon.Not. Roy.Astron.Soc, Vol.335 (3), pp.843-852, 2002.

8) Koshizuka, S., Oka, Y.: Moving-particle semi-implicit method for fragmentation of incompressible fluids, $\mathrm{Nucl}$. Sci. Eng.,Vol. 123, pp. 421-434, 1996.

9) Koshizuka, S., Nobe, A., Oka Y.: Numerical analysis of breaking waves using the moving particle semi-implicit method, Int. J. Numer. Methods Fluids., Vol.26, pp.751769. 1998.

10) Khayyer, A., Gotoh, H.: Modified Moving Particle Semi-implicit methods for the prediction of $2 \mathrm{D}$ wave impact pressure, Coastal Eng., Vol.56 (4), pp.419-440, 2009.

11) Gotoh, H., Sakai, T.: Key Issues in the Particle Method for Computation of Wave Breaking, Coastal Eng., Vol.53 (2-3), pp.171-179, 2006.

12) K. Shibata, S. Koshizuka.: Numerical analysis of shipping water impact on a deck using a particle method, Ocean Engineering., Vol.34 (3-4), pp. 585-593, 2007.

13) S. Koshizuka, M.S. Song, Y. Oka.: A particle method for three-dimensional elastic analysis, in: Proceedings of the Sixth World Congress on Computational Mechanics., Beijing, September, 5-10, 2004.

14) Ikari, H., Gotoh, H., Sakai, T., Tanioka, H.: Analysis of impact wave pressure by particle method for compressible fluid, Annual Journal of Coastal Engineering JSCE 52 (1), pp.731-735 (in Japanese), 2005.

15) HIBI S., YABUSHITA K.: A Study on Reduction of Unusual Pressure Fluctuation of MPS Method, Journal of the Kansai Society of Naval Architects., Japan (241), 125-131, 03-25 (in Japanese), 2004.

16) Khayyer, A. \& Gotoh, H.: Enhancement of stability and accuracy of the moving particle semi-implicit method, $J$. Comput. Phys., Vol. 230, Issue 8, pp. 3093-3118, 2011.

17) Liu GR, Liu MB.: Smoothed particle hydrodynamics: a meshfree particle method, World Scientific; 2003.

18) Monaghan J.J., Lattanzio JC.: A refined particle method for astrophysical problems, Astron Astrophys., 149:135, 1985.

19) M.B. Liu - G.R. Liu.: Smoothed Particle Hydrodynamics (SPH): an Overviewand Recent Developments, Arch Comput Methods Eng., Vol.17, pp. 25-76, 2010.

20) Flebbe, O., Muenzel, S., Herold, H., Riffert, H., \& Ruder, H.: Smoothed Particle Hydrodynamics: Physical Viscosity and the Simulation of Accretion Disks, The Astrophysical Journal, Vol. 431, no. 2, pt. 1, pp. 754-760,1994.

21) Dalrymple, R.A., Knio, O.: SPH modelling of water waves, Proc. Coastal Dynamics, pp.779-787, Sweden, 2001.

22) S. Koshizuka, H. Tamko, Y. Oka. : A particle method for incompressible viscous flow with fluid fragmentation, Comp. FluidDyn. J., vol. 4 (1), pp. 29-46, 1995.

23) Janosi, I. M., Jan, D., Szabo, K. G., and Tel, T.: Turbulent drag reduction in dam-break flows, Exp. Fluids, Vol. 37(2), pp. 219-229, 2004.

24) Monaghan, J. J.: On the problem of penetration in particle methods, J. Comput. Phys., Vol. 82, pp. 1-15, 1989.

25) James C. Lombardi, Alison Sills, Frederic A. Rasio, Stuart L. Shapiro.: Tests of Spurious Transport in Smoothed Particle Hydrodynamics, J. Comput. Phys., Vol.152, pp.687-735, 1999.

26) Shakibaeinia, A., Jin, Y.C.: A weakly compressible MPS method for modeling of open-boundary free-surface flow,International J. Numer. Methods Fluids., Vol 63(10) ,pp. 1208-1232, 2009. 